
\title{
25 Research Square \\ Ocular Vogt-Koyanagi-Harada: Is it the era of diagnosis by OCT?
}

Khaled Elubous ( $\nabla$ khalidalebous@hotmail.com )

The University of Jordan https://orcid.org/0000-0002-8689-2585

Mohammed S. Farah

The University of Jordan

Ahmed N. Shokry

The University of Jordan

Mohammed Abu-Ameereh

The University of Jordan

\section{Case Report}

Keywords: Vogt-Koyanagi-Harada syndrome, optical coherence tomography, fluorescein angiography, sensory retinal detachment

Posted Date: June 29th, 2021

DOI: https://doi.org/10.21203/rs.3.rs-654480/v1

License: (c) (1) This work is licensed under a Creative Commons Attribution 4.0 International License. Read Full License 


\section{Abstract}

\section{PURPOSE}

to report optical coherence tomography (OCT) changes of the retina during acute and recovery phases in a patient with atypical Vogt-Koyanagi-Harada (VKH) disease presented for the first time with ocular symptoms and role of (OCT) in the diagnosis, following and assessing response to treatment.

\section{Methods}

Ophthalmologic examination, laboratory evaluation, fluorescein angiography, and spectral-domain optical coherence tomography performed on a twenty-four-year-old female presented with a bilateral blurring of vision of 3 days.

\section{Results}

OCT disclosed multifocal subretinal lobulated cystic fluid collections with thickening and hyperreflectance in outer retinal layers. Decreased retinal thickness and resolving subretinal fluid collections were seen after commencing treatment by systemic corticosteroids.

\section{Conclusion}

OCT is a very sensitive tool to diagnose and monitor the highly detailed retinal changes during the $\mathrm{VKH}$ course especially if presented primarily with ocular symptoms

\section{Introduction}

Vogt-Koyanagi-Harada (VKH) disease is a chronic bilateral granulomatous panuveitis often associated with extraocular manifestations in the central nervous, auditory and integumentary systems.[1] The inflammatory manifestations are thought to be an autoimmune reaction directed against proteins related to stromal choroidal melanocytes. The initial inflammatory events, therefore, occur at the level of the choroidal stroma and adjacent structures, such as the retinal pigment epithelium (RPE) and the retina, are involved only secondarily.[2] It is usually presented with prodromal symptoms followed by an ocular stage then neurological and skin changes.

Ocular manifestations of VKH disease are characterized by bilateral inflammatory diffuse choroiditis and exudative serous retinal detachments, responsive to treatment with high-dose systemic corticosteroids.[3]

Fluorescein angiography (FA) in VKH patients reveals multiple dot or placoid points of leakage at the level of retinal pigment epithelium (RPE) in the early phase, and multifocal dye pooling under a detached sensory retina or RPE in the late phase.[4] 
Optical coherence tomography (OCT) is a non-invasive, non-contact, transpupillary imaging technology. It is based on low coherence interferometry that provides optical cross-sectional images of the eye.[1] It allows the measurement of retinal thickness from tomograms by means of computer image-processing techniques.[1] Spectral-domain OCT (SD-OCT) allows better identification of individual retinal layers and provides much more needed clinicopathologic information than time-domain OCT.[2]

We aim to describe the use of SD-OCT to measure and monitor retinal changes during the acute and convalescence phases of ocular VKH disease.

\section{Materials And Methods}

Ophthalmic evaluation upon initial presentation included best-corrected visual acuity (BCVA), intraocular pressure, slit-lamp examination, funduscopy using slit-lamp non-contact lenses, Ishihara color plate tests, FA and spectral-domain OCT. A comprehensive rheumatological, gynecological, dermatological, and otolaryngological screening was also performed.

\section{Case Report}

24 years old female patient presented to our ophthalmology clinic with a 3 days history of bilateral blurring of vision of sudden onset. Upon detailed ophthalmic questioning, there were no other ocular complaints. The blurring of vision was progressive over the 3 days. There were no associated redness nor discharge and no complaints of flashes or floaters. There was no significant past ophthalmic history, no history of eye trauma or eye surgeries. Past medical and surgical histories were also insignificant. Past drug history showed that the patient was on oral contraceptive pills for the past 2 years. Upon further questioning, we didn't find any systemic complaints such as tinnitus or hearing loss, no neurological symptoms, and no evidence of any dermatological abnormalities

On detailed clinical examination, the patient's visual acuity was 0.2 in her right eye and 0.05 in the left eye. There was no improvement in visual acuity with refraction. There was no relative afferent pupillary defect. Ishihara testing revealed 10\13 in the right eye and 0/13 in the left eye. On slit-lamp biomicroscopy, the anterior segment was examined. The eye was quiet and there were no signs of anterior segment inflammation. Intraocular pressure was $8 \mathrm{mmHg}$ in the right eye and $7 \mathrm{mmHg}$ in the left.

Posterior segment examination revealed bilateral multiple deep creamy white to yellow lesions that were distributed in a scattered patchy pattern all over the posterior pole (figure-1). Lesions were very similar in shape and pattern in both eyes. The examination also revealed multiple focal areas of neurosensory elevations or cysts of different sizes, the largest being at the macula along with striations of the neurosensory layer. These areas were also seen in both eyes and were comparable. There was no vitritis nor vitreous opacities, no papillitis, and no apparent vasculitis.

Ophthalmic investigations were ordered; FA and SD-OCT were done. FA revealed abnormal hyperfluorescent points scattered through the posterior pole in the early phases that increased in intensity 
and size in the late phases representing areas of subretinal pooling. Some of the hyperfluorescent points only increased in intensity representing pooling under the retinal pigment epithelial (figure-2). OCT macula revealed multiple areas of subretinal and intraretinal fluid accumulations along with multifocal atypical wide-angle neurosensory retinal detachments. Central macular thickness reached $922 \mu \mathrm{m}$ in the right eye and $819 \mu \mathrm{m}$ in the left. Another significant finding in the OCT was the appearance of the intraretinal and subretinal fluid where it appeared to be heterogeneous and turbid along with strands in the fluid that was hyperintense in signal (figure-3). The above examination and investigations went with multifocal choroiditis involving the posterior pole along with multiple sensory neural detachments. At the top of our differential diagnosis was atypical or ocular VKH.

The patient was admitted for further evaluation. Dermatology, Rheumatology as well as ear, nose, and throat (ENT) consults were ordered that all come without any abnormality to be considered. The patient was started on oral prednisolone $80 \mathrm{mg}$ per day. After $48 \mathrm{hrs}$ of starting oral steroids vision improved; O.D, 0.4 and $0 . S, 0.25$. The patient was discharged then and was kept on the same dose of oral steroids. 2 days later she was seen in the clinic with further improvement in vision reaching 0.5 in 0.D and 0.3 in 0.S. A new SD-OCT was done then which showed a remarkable decrease in retinal thickness in both eyes, resolution of subretinal strands and the subretinal fluid becomes clearer with flattening of neurosensory retinal detachments (figure-4) on which the decision had been made to start tapering the treatment slowly over a period of 6 weeks. The patient was seen 6 weeks after the primary visit in the clinic with a dramatic improvement in vision reaching $0.8 \mathrm{O} . \mathrm{U}$

\section{Discussion}

Vogt-Koyanagi-Harada (VKH) disease is an autoimmune multisystem disorder directed against melanocytes. Acute ocular inflammation occurs as a bilateral granulomatous panuveitis characterized by bilateral diffuse choroiditis and prominent multifocal serous exudative retinal detachments. It is often associated with extraocular manifestations in the central nervous and integumentary systems. $[4,5]$

As a new non-invasive diagnostic technique, OCT has become a method of choice to follow a number of macular diseases. Its reproducibility to measure retinal thickness in healthy subjects or in diabetic patients with macular edema has been demonstrated. OCT gives a high resolution of the images, with a longitudinal resolution of 10 microns.[1]

Our case represents an unusual form of VKH in which the initial presentation was ocular i.e. sudden bilateral loss of vision with no other suggestive systemic symptoms or signs that can aid in the diagnosis. The visual acuity was so markedly reduced in the left eye more than the right which was parallel to a more increased retinal thickness in the left eye as shown by OCT images. A beneficial effect of corticosteroids was noticeable within days in the OCT images that were equivalent to the improvement in visual acuity for which treatment had been tapered slowly.

The (SD-OCT) of our patient showed significant changes in the retina during the acute stage involving multiloculated, cystic collections of serous fluid mixed with membranous structures under the sensory 
retina and the RPE, hyper-reflectance in outer retinal layers, intraretinal edema, subretinal septa, and no RPE folds had been noticed in contrary to what has been found by Kato $Y$ et al who concluded that the detection of the folds of the RPE on OCT is a simple and effective method to help diagnose VKH disease at its acute stage which does not require pupil dilation.[5] The OCT of the patient did not show also choroidal folds which appeared in the OCT of one patient reviewed by Shinoda K.[6]

The tomographic features of serous retinal detachment (SRD) in eyes with VKH disease have been investigated in detail using OCT.[4] Yamaguchi et al. detected a membrane on the RPE and subretinal septa ( $90 \%$ sensitivity for $\mathrm{VKH}$ ) which disappeared immediately after treatment, and they assumed that the membrane was composed of inflammatory products such as fibrin.[7]. Other OCT studies of eyes with VKH disease have shown an SRD during the acute and/or convalescence stages [1], and Yamanaka et al. showed a significant decrease in the height of the SRD immediately after the first and second intravenous corticosteroid injections.[8] The same significant improvement in the retinal structure was seen in our patient after 2 days of oral, not intravenous corticosteroids. Yamamoto et al prove that SD-OCT is able to demonstrate characteristics of VKH that no other study to date has detected.[2]

Ikewaki et al in their study mentioned that OCT parameters can be a reliable non-invasive real-time indicator of the severity of the inflammation and the effectiveness of the treatment in VKH and these parameters can be as important as the visual acuity. They also indicate that by using OCT, dynamic changes in the macular morphology reflecting the effect of the treatment can be monitored quantitatively and precisely and this may guide when the tapering of the dosage of steroids should begin and end.[4] Regarding our patient, the decision to taper the treatment depended on the significant improvement in retinal structure seen in OCT as mentioned in this study.

Parc et al studied the OCT changes in the acute and chronic phases of VKH disease and demonstrated an atypical serous retinal detachment with the presence of intraretinal edema. The latter manifested as a hyporeflective area corresponding to liquid located between different layers of the neurosensory retina. They also notice retinal pigment epithelium (RPE) thickening corresponding to RPE hypertrophy and fibrosis.[1] Nakai $\mathrm{K}$ et al concluded that significant choroidal thickness changes underlie VKH disease, which progresses over time, and their objective measurement (OCT) may be useful for longitudinal evaluation of VKH activity.[9] Another finding by Theodoro da Silva F et al was that patients with VKH and long-standing disease had thinner choroids when compared to controls.[10]

\section{Declarations}

\section{Competing interests}

The authors declare that they have no competing interests.

Informed written consent was obtained from the patient.

\section{References}


1. Parc C, Guenoun JM, Dhote R, Brezin A (2005) Optical Coherence Tomography in the acute and chronic phases of Vogt Koyanagi Harada Disease. Ocular Immunology and Inflammation; 13:225227

2. Yamamoto $M$, Nishijima K, Nakamura $M$, Yoshimura N (2011) Inner Retinal Changes in Acute-Phase VogtKoyanagi-Harada Disease Measured by Enhanced Spectral Domain Optical Coherence Tomography. Jpn J Ophthalmol;55:1-6

3. Rubsamen PE, Gass DM(1991) Vogt-Koyanagi-Harada syndrome. Arch Ophthalmol; 109:682-687.

4. Ikewaki J, Kimoto K, Choshi T, Nagata M et al (2011) Optical coherence tomographic assessment of dynamic macular changes in patients with Vogt-Koyanagi-Harada disease. Int Ophthalmol; 31:913

5. Kato Y, Yamamoto Y, Tabuchi H et al (2013) Retinal pigment epithelium folds as a diagnostic finding of Vogt-Koyanagi-Harada disease. Jpn J Ophthalmol.; 57:90-94.

6. Shinoda K, Imamura A, Matsumoto C, Mizota A (2012) The detection of the folds of the RPE on OCT is a simple and effective method to help diagnose VKH disease at its acute stage which does not require pupil dilation. Graefes Arch Clin Exp Ophthalmol; 250:1399-1402

7. Yamaguchi Y, Otani T, Kishi S (2007) Tomographic features of serous retinal detachment with multilobular dye pooling in acute Vogt-Koyanagi-Harada disease. Am J Ophthalmol 144:260-265

8. Yamanaka E, Ohguro N, Yamamoto S, Nakagawa Y, Imoto Y, Tano Y (2002) Evaluation of pulse corticosteroid therapy for Vogt-Koyanagi-Harada disease assessed by optical coherence tomography. Am J Ophthalmol 134:454-456

9. Nakai K, Gomi F, Ikuno Y, Yasuno Y et al (2012) Choroidal observations in Vogt-Koyanagi-Harada disease using high-penetration optical coherence tomography. Graefes Arch Clin Exp Ophthalmol 250:1089-1095

10. Theodoro da Silva F, Sakata VM, Nakashima A, Hirata CE et al (2013) Enhanced depth imaging optical coherence tomography in long-standing Vogt-Koyanagi-Harada disease. Br J Ophthalmol; $97,70-74$

\section{Figures}
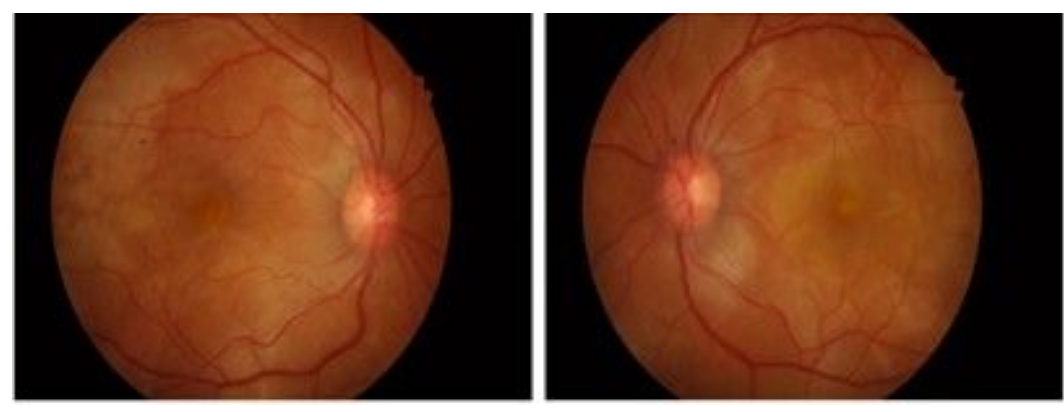

\section{Figure 1}


Figure 1. Color fundus photographs, right (OD) and left (OS) eyes
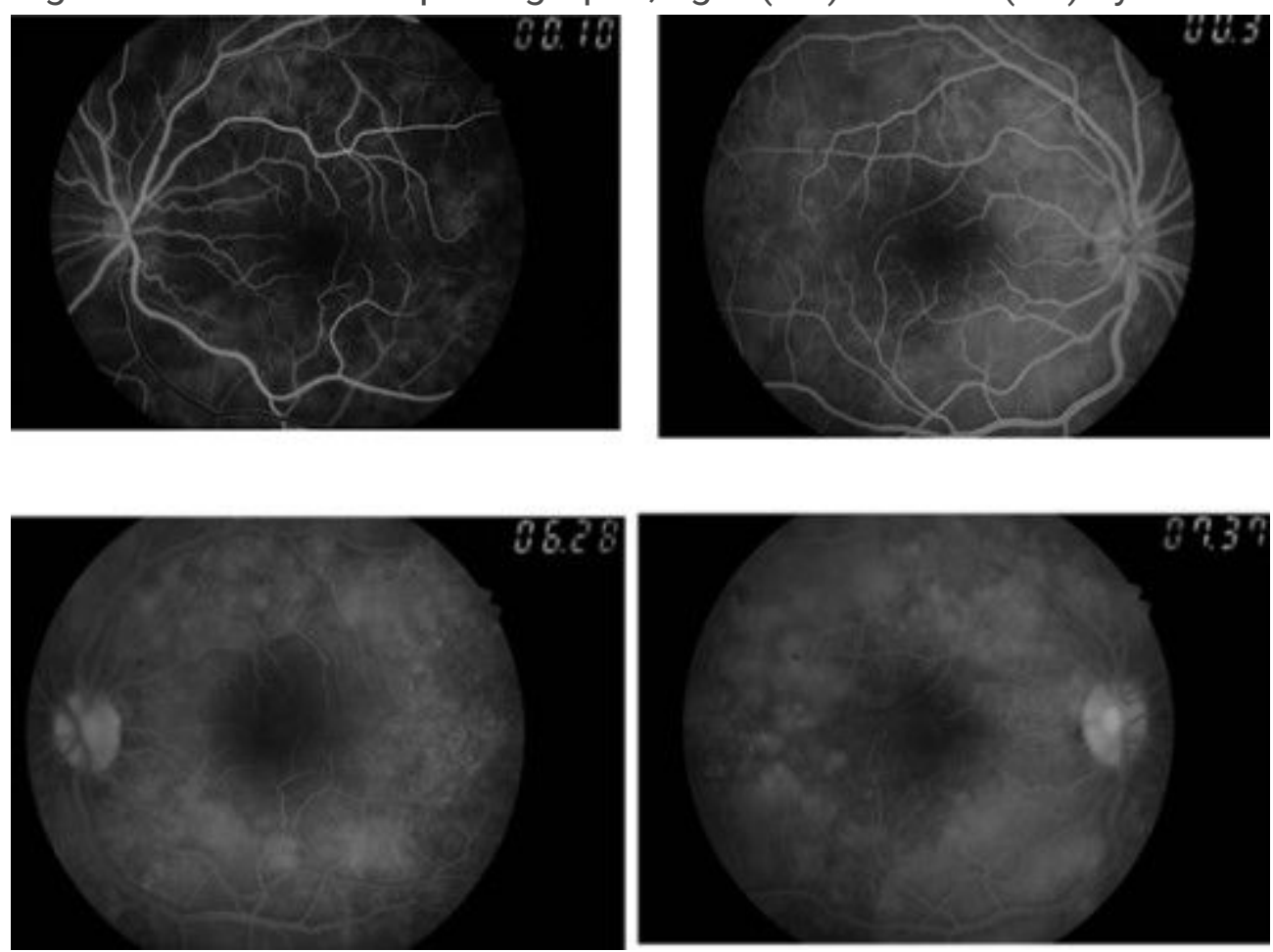

Figure 2

figure 2. Fundus fluorescein angiography 

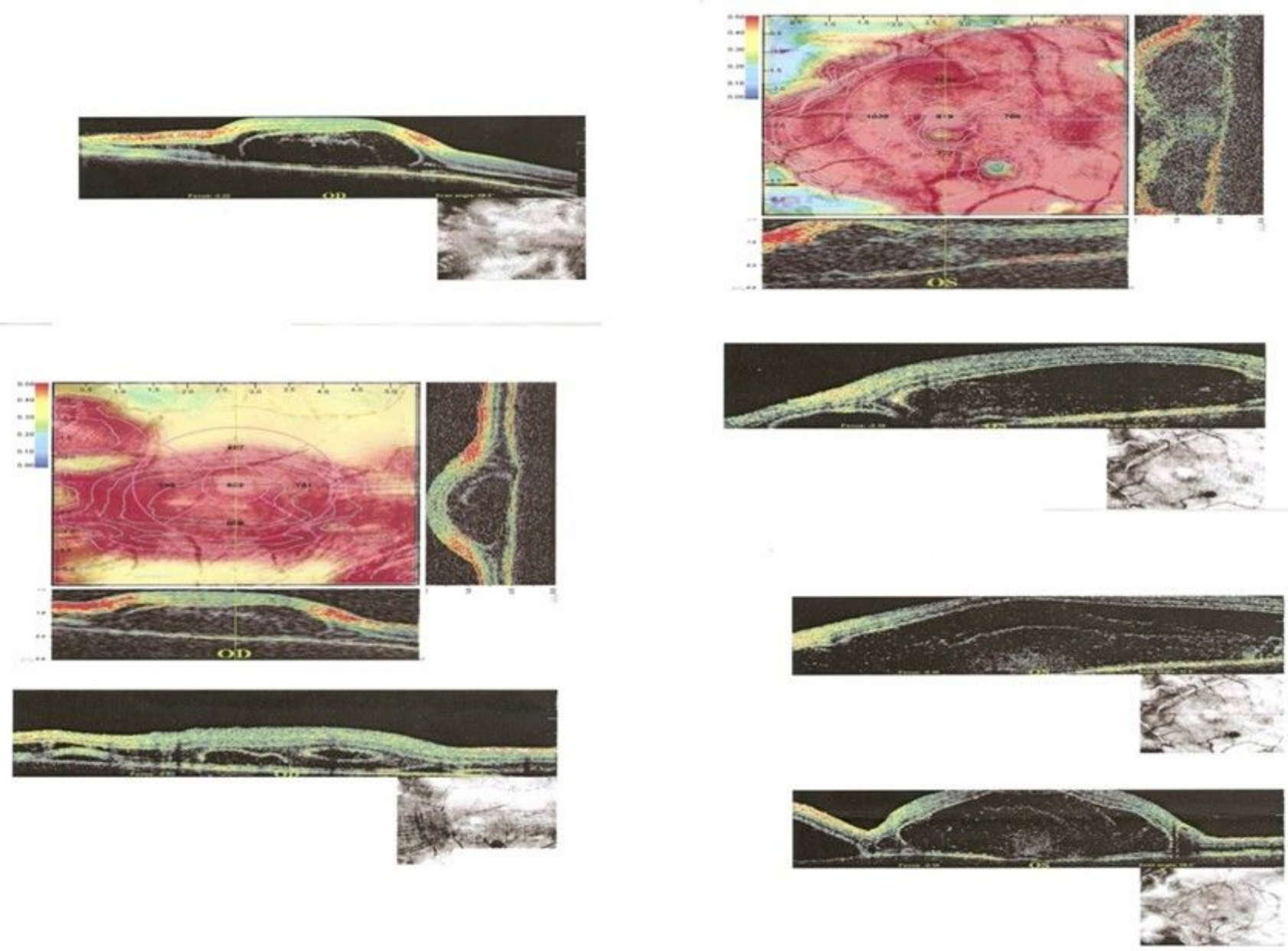

Figure 3

Figure 3. SD-OCT before treatment showing right (OD) and left (OS) eyes 

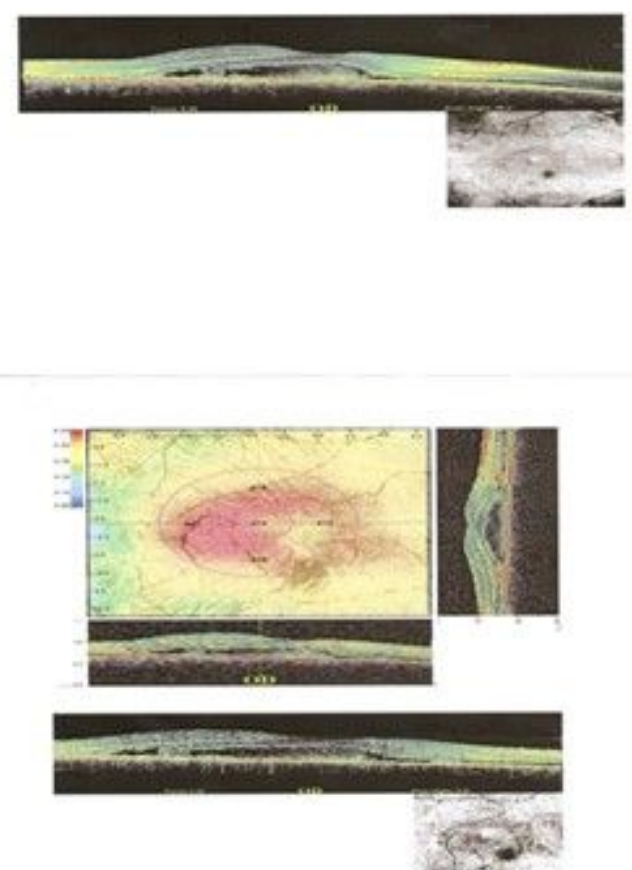
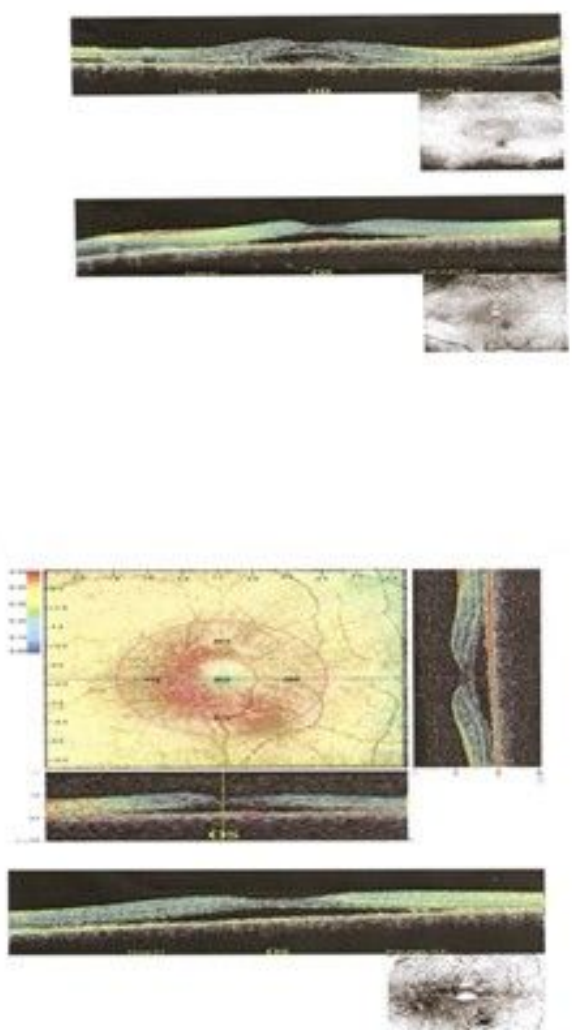

Figure 4

Figure 4. SD-OCT same patient after initiation of treatment 